\title{
Experiencias para una Alfabetización Científica que Promueva la Justicia Ambiental en Distintos Niveles Educativos
}

\author{
Experiences for a Scientific Literacy that Promotes \\ Environmental Justice at Different Educational Levels
}

\author{
José Manuel Pérez Martín * \\ Beatriz Bravo Torija
}

Universidad Autónoma de Madrid, España

\begin{abstract}
La situación medioambiental en el siglo XXI refleja un problema complejo que nos afecta de manera desigual. La educación ambiental requiere de un enfoque sociocientífico que amplíe su perspectiva para promover la comprensión de las situaciones reales, y conducir a un posicionamiento fundamentado y crítico de la sociedad para la justicia ambiental. Para conseguirlo, necesitamos una alfabetización científica desde un enfoque Científico, Tecnológico, Social y Ambiental (CTSA). Con este fin, realizamos tres intervenciones de aula en diferentes niveles educativos estructuradas en tres fases: a) distribución de información conceptual; b) reflexión a través del pensamiento crítico, y c) elaboración de propuestas para solucionar los problemas tratados. En cada propuesta, se presentan contenidos relativos a problemáticas de contaminación y se relacionaron con los problemas de salud humana y ambiental. El análisis de estas actividades se realizó a partir de las notas de los comentarios más relevantes, dibujos y/o un trabajo. Todo ello nos indica que su diseño es adecuado para enlazar la distribución de conocimientos, con la participación en la toma de decisiones, elementos clave para el desarrollo de una educación para la justicia ambiental.
\end{abstract}

Descriptores: Alfabetización; Justicia social; Docente; Educación ambiental; Ciencia.

The environmental situation in the 21 st century reflects a complex problem that affects us unequally. Environmental education requires a socio-scientific approach that broadens this perspective in order to promote the understanding of real situations, and leads to an informed and critical positioning of society for Environmental Justice. To do so, we need a scientific literacy from a Scientific, Technological, Social and Environmental (STSE) approach. To this aim, we carried out three classroom involvements in different educational levels structured in three phases: (a) Distribution of little known conceptual information about the problem; (b) reflection about it the promotion of critical thinking, and c) preparation of proposals to solve the problem. In each proposal, content related to pollution issues was presented and related to human and environmental health problems. The analysis of these activities was carried out taking into account the most relevant notes from conversation, drawings and/or class works. All data indicates that their design is adequate to link the knowledge distribution with students' participation in decision-making: key elements for the development of an education for environmental justice.

Keywords: Literacy; Social justice; Teachers; Environmental education; Science.

*Contacto: josemanuel.perez@uam.es

ISSN: 2254-3139

www.rinace.net/riejs/

revistas.uam.es/riejs
Recibido: $\quad 16$ de septiembre 2017

$1^{\text {a }}$ Evaluación: 7 de diciembre 2017

$2^{\text {a }}$ Evaluación: 29 de diciembre 2017

Aceptado: $\quad 7$ de enero 2018 


\section{Introducción}

Tradicionalmente la educación ambiental ha tenido un enfoque reducido a la presentación de problemas medioambientales y al planteamiento de situaciones convencionales, no existiendo una definición concreta de las competencias a trabajar en su enseñanza (Cebrián y Junyent, 2014). Sin embargo, hace tiempo que se propone que para comprender la problemática del medio ambiente actual se requiere de una Educación Ambiental enmarcada en el paradigma de la complejidad (Bonil, Junyent y Pujol, 2010). En este contexto, se debe entender que la complejidad hace referencia a la dificultad de interpretación y previsión del riesgo que conllevan estas situaciones y a la incertidumbre e imprevisibilidad de las intervenciones que se realizan. En definitiva, a la toma de conciencia de que sus límites no están bien definidos. Esto se debe a que las problemáticas ambientales implican diferentes áreas de conocimiento (sociales, económicos, culturales y científicos, entre otros), existiendo múltiples estructuras de enlace y relaciones entre conceptos y procesos (Mogesen y Mayer, 2009).

Dada la necesidad de que los ciudadanos estén capacitados para la toma de decisiones en las sociedades democráticas modernas, entre las que se encuentran aquellas relacionadas con el medio ambiente y la actuación del ser humano en él, es imprescindible trabajar en las aulas la alfabetización científica con un enfoque denominado Ciencia, Tecnología, Sociedad y Ambiente (CTSA). Lo que promueve no solo considerar las problemáticas ambientales desde una perspectiva multifactorial (Solís-Espallargas y ValderramaHernández, 2015), sino también desarrollar destrezas que fomenten la autonomía en el aprendizaje del alumnado. Esto requiere superar una enseñanza dogmática de las ciencias, formando ciudadanos capaces de comprender las relaciones existentes entre el desarrollo científico-tecnológico y sus consecuencias para la sociedad.

Sin embargo, los programas formativos de corte ambiental se centran en la presentación de problemas globales y soluciones convencionales, como el reciclaje. Además, en algunas ocasiones, las soluciones sugeridas por el alumno no suponen cambios en su conducta, ya que la situación presentada es lejana y le hace poco partícipe de la toma de medidas concretas.

Por el contrario, las propuestas presentadas en este trabajo se refieren a problemas próximos, dado que ocurren en su entorno local, y las acciones que ellos mismos sugieren, suponen cambios en la situación tratada. Estas intervenciones se realizan en aulas de diferentes niveles educativos, y los temas tratados giran en torno a la presencia de residuos en el medio acuático y la cadena trófica, y sus efectos sobre la salud humana y ambiental. Todas ellas persiguen la distribución de contenidos poco conocidos como la presencia de contaminantes ambientales de origen doméstico (residuos sólidos, plastificantes, fármacos o productos de cuidado personal) en aguas superficiales, las vías de incorporación, sus riesgos para el ecosistema; la reflexión sobre el tema, así como el fomento del desarrollo de acciones en las que los alumnos puedan intervenir, enmarcándose en una educación para la justicia ambiental. 


\section{Fundamentación teórica}

\subsection{Justicia ambiental}

La visión ambientalista de protección del medio natural tiene una larga tradición, con intensa actividad durante la segunda mitad del siglo XIX. Las propuestas para su conservación han estado relacionadas siempre con acciones reivindicativas de presentación de la problemática y posibles soluciones para su restitución. En paralelo, desde finales del siglo Xx aparece en Estados Unidos un movimiento que propone un nuevo enfoque en la percepción de este tema, la justicia ambiental. Dicho enfoque considera que el aprovechamiento de los recursos y fuentes de energía, así como los riesgos que generan los residuos producidos, no se distribuyen equitativamente entre los distintos grupos humanos, siendo las clases sociales bajas las más perjudicadas (Ramírez, Galindo y Contreras, 2015).

En ese punto es conveniente destacar que el movimiento de justicia ambiental entronca con el de justicia social, y al igual que éste, no es fácil de definir (Arriaga y Pardo, 2011). Según Murillo y Hernández-Castilla (2011), la justicia social propone como idea fundamental la necesidad de que exista equidad entre personas en el acceso a los bienes y recursos, citando tres dominios interrelacionados: la distribución de los bienes y recursos, entre ellos el conocimiento; el reconocimiento de la diversidad, el respeto y dignidad de las personas y la participación en la toma de decisiones. Por ello, es interesante el enfoque que la justicia social le aporta al movimiento ambientalista, incompleto sin estos componentes (Arriaga y Pardo, 2011) dado que, incorporar cuestiones de equidad social y económica, conduce a abordar el problema con un enfoque más integral de la realidad (Agyerman, 2007), el de justicia ambiental (Grass, 1995).

La justicia ambiental tiene como objetivo la lucha contra la distribución desigual de riesgos ambientales para la salud, del uso de los recursos naturales y de la participación en la toma de decisiones (Ramírez, Galindo y Contreras, 2015; Sarokin y Schulkin, 1994). Incluso la Agencia de Protección Medioambiental de Estados Unidos comparte esta idea (Wende, 2013). Sin embargo, algunos autores consideran que esta labor hay que hacerla desde un paradigma más amplio, como la reconsideración de los valores de uso de los recursos naturales (Dobson, 1998; Fuente, Tagle y Hernández, 2015), el uso ético y responsable de dichos recursos (Ponce, 2012; Riechman, 2003), o la conservación del medio ambiente (Hiskes, 2006).

Teniendo en cuenta dichos factores, la evolución del movimiento por la justicia ambiental culmina con el establecimiento de 17 principios (Arriaga y Pardo, 2011), entre los que destacamos, la exigencia del cese de producción de toxinas (6), el derecho a vivir y trabajar en un ambiente saludable (8), el derecho a un medio ambiente urbano sano (12), la implementación estricta de los principios de información y consentimiento (13), la promoción entre las generaciones futuras de una educación con énfasis en cuestiones sociales y medioambientales (16), y la minimización del consumo de recursos naturales y generación de residuos (17).

Para cumplir estos principios necesitamos una educación para la justicia ambiental, a lo que, desde las ciencias, podría ayudar la adquisición por parte del alumnado de una adecuada alfabetización científica, que le capacite para comprender los problemas científicos y tecnológicos actuales, sus consecuencias, y su implicación en ellos. Por esta 
razón, consideramos que puede ser de utilidad trabajar las ciencias, y en particular el medio ambiente, desde un enfoque CTSA.

\subsection{Alfabetización científica desde un enfoque CTSA}

Con respecto a qué se considera Educación CTSA, en la literatura encontramos que no existe una definición única (Aikenhead, 2003). Dado el interés de este trabajo, consideramos aquéllas en las que los autores señalan la necesidad de una formación en CTS que persigue formar ciudadanos alfabetizados científicamente. Santos y Mortimer (2001) hacen hincapié en que una educación CTS debe reconocer la importancia de una alfabetización científica y tecnológica para la toma de decisiones responsables en asuntos controvertidos que implican a la ciencia y la sociedad. Vilches et al. (2008) completan esta definición conectando la educación CTS con el desarrollo de una educación para un futuro sostenible, reconociendo la necesidad de preparar a una sociedad consciente de los problemas del planeta, capaz de adoptar medidas para superarlos, reformulando el término CTS COMO CTSA.

Este tipo de formación permite vincular discusiones externas a la educación científica con las prácticas escolares (Strieder, 2012), promoviendo la existencia de una ciencia y una tecnología que va más allá de su visión como solucionadora de los problemas, reconociendo sus incertezas y limitaciones. Esto conlleva alejarse de una idea cerrada y neutral del desarrollo científico y tecnológico, reconociendo que el rumbo que ha tomado, y está tomando, se encuentra marcado por una sociedad llena de desequilibrios políticos, económicos, culturales y ambientales (Strieder, Bravo-Torija y Gil-Quílez, 2017).

Entre los trabajos que han tratado la ciencia y la tecnología desde esta perspectiva, encontramos que el grado de implicación del alumnado en ellos ha sido muy variable. Desde los que presentan el problema al alumnado, proporcionándoles conocimientos sobre el mismo, pero sin considerar su actuación en él, como los de Viegas y Santos (2012) o Ezquerra y Fernández (2014); hasta los que desde un inicio le solicita que tome conciencia de que su actuación en el medio tiene un coste, y que, como parte de la sociedad, ha de intervenir en el proceso de producción e implementación de los productos científicotecnológicos (Chrispino et al., 2013; Teixeira y Formenton, 2012). Aunque estos últimos son los que presentan un mayor interés desde el punto de vista de la educación para la justicia ambiental, la mayoría de estos estudios se caracterizan por ser trabajos teóricos, que discuten cómo debería ser una adecuada educación CTSA e incluso hacen propuesta para el aula, pero todavía no han llegado a implementarse (Strieder, Bravo-Torija y GilQuílez, 2017). Este objetivo es el que persiguen las propuestas que se presentan a continuación, no sólo informar al alumnado acerca del problema, sino hacerle partícipe, pidiéndoles soluciones, e implicación por su parte, en la medida de lo posible.

\section{Metodología}

El objetivo general del trabajo es presentar acciones formativas sobre CTSA para fomentar actitudes de justicia ambiental en distintos niveles educativos, analizando qué acciones son capaces de desarrollar los alumnos en ellas.

La primera propuesta que se describe se realiza en Educación Infantil, ya que según diferentes autores existe una carencia de actividades para este colectivo (Davis, 2009; Elliott, 2010; García-Esteban y Murga-Menoyo, 2015). El trabajo continúa con una experiencia con alumnos de $2^{\circ}$ de Bachillerato (17-18 años). En este caso, tanto en la 
Educación Secundaria Obligatoria como en el Bachillerato tienen los contenidos de la Educación Ambiental bien definidos. Sin embargo, se considera que las actividades suelen quedarse en un ámbito superficial, restringiéndose a aspectos informativos (Novo, 2005). Por último, también se lleva a cabo una intervención con estudiantes universitarios del Grado en Educación Infantil, que promueve acciones educativas para la justicia ambiental, ya que son profesionales que demuestran interés por el medio ambiente, pero presentan carencias formativas respecto a este conocimiento (Cantó, 2016).

En cuanto a la ausencia de propuestas para el nivel de Educación Primaria, hay que decir que no ha llegado a ser implementada, y aunque generalmente se considera que es una etapa en la que existen una gran cantidad de materiales docentes (Vílchez López, 2005), sigue existiendo una deficiencia en aquellos que promueven la adquisición de destrezas científicas por parte de este alumnado (Martínez y García, 2003).

La herramienta empleada para recopilar los resultados de cada experiencia fue un registro de los comentarios más relevantes que iban surgiendo a partir de un conjunto de preguntas intercaladas en el relato de la presentación. Estas preguntas se denominan preguntas mediadoras, preguntas para pensar (del inglés, thought-provoking questions) o preguntas que ayudan a aprender (Andersson y Gullberg, 2014; Márquez y Roca, 2006; Roca, Bonil y Pujol, 2005), y su utilidad ya ha sido demostrada en secuencias didácticas de relato y reflexión en nuestro grupo (García González y Pérez Martín, 2016), desarrollándose entre los alumnos interpretaciones más completas de la realidad, y con ellas el juicio crítico.

En el caso de la experiencia de Educación Infantil y en la desarrollada con maestros en formación, se recogieron datos a partir de dos instrumentos de evaluación de aula, y describimos algunos de los resultados a modo de ejemplo de forma cualitativa y no sistemática. En el primer caso, seis meses después de la intervención (final de curso, junio) se les solicitó un dibujo sobre la temática del mar y la contaminación. Este largo plazo nos permite intuir si se produce retención de lo aprendido y, en cierta medida, refleja el impacto causado en el proceso de enseñanza-aprendizaje. El dibujo infantil es una herramienta de análisis muy potente para realizar estudios en cualquier etapa educativa (Goodnow, 1979), pero resulta más trascendente con alumnos cuyo dominio de la escritura es limitado o nulo. En este sentido, las bondades de esta herramienta no pueden ser olvidadas en la enseñanza de las Ciencias (Ainsworth, Prain y Tyler, 2011). En cualquier caso, sí es muy recomendable que los alumnos describan el dibujo de forma oral, y que el adulto recoja las descripciones de los detalles, como se muestra en nuestros resultados para el ejemplo presentado. En la experiencia llevada a cabo con los maestros en formación, se requirió la redacción de una circular escolar dirigida a las familias de los alumnos de Educación Infantil en la que se explicase la problemática presentada en la sesión de intervención, así como información complementaria y una reflexión crítica con posibles acciones cotidianas. Todo ello, se muestra como ejemplo en fragmentos representativos de algunos trabajos.

Una vez descrita cada propuesta, se presentan las acciones concretas que realizan los estudiantes en cada una de ellas. Para hacerlo, se toma como referencia la propuesta de Medir, Heras y Magin (2016). Entre las acciones señaladas por estos autores, destacan tanto la adquisición de conocimientos, procedimientos y actitudes, como la búsqueda de información, el cuestionamiento de los conocimientos asumidos o el planteamiento de preguntas. Todas ellas clasificables en acciones de distribución de contenidos, reflexión de la situación y toma de posición con acciones concretas (cuadro 1). 
Cuadro 1. Acciones a realizar en las propuestas

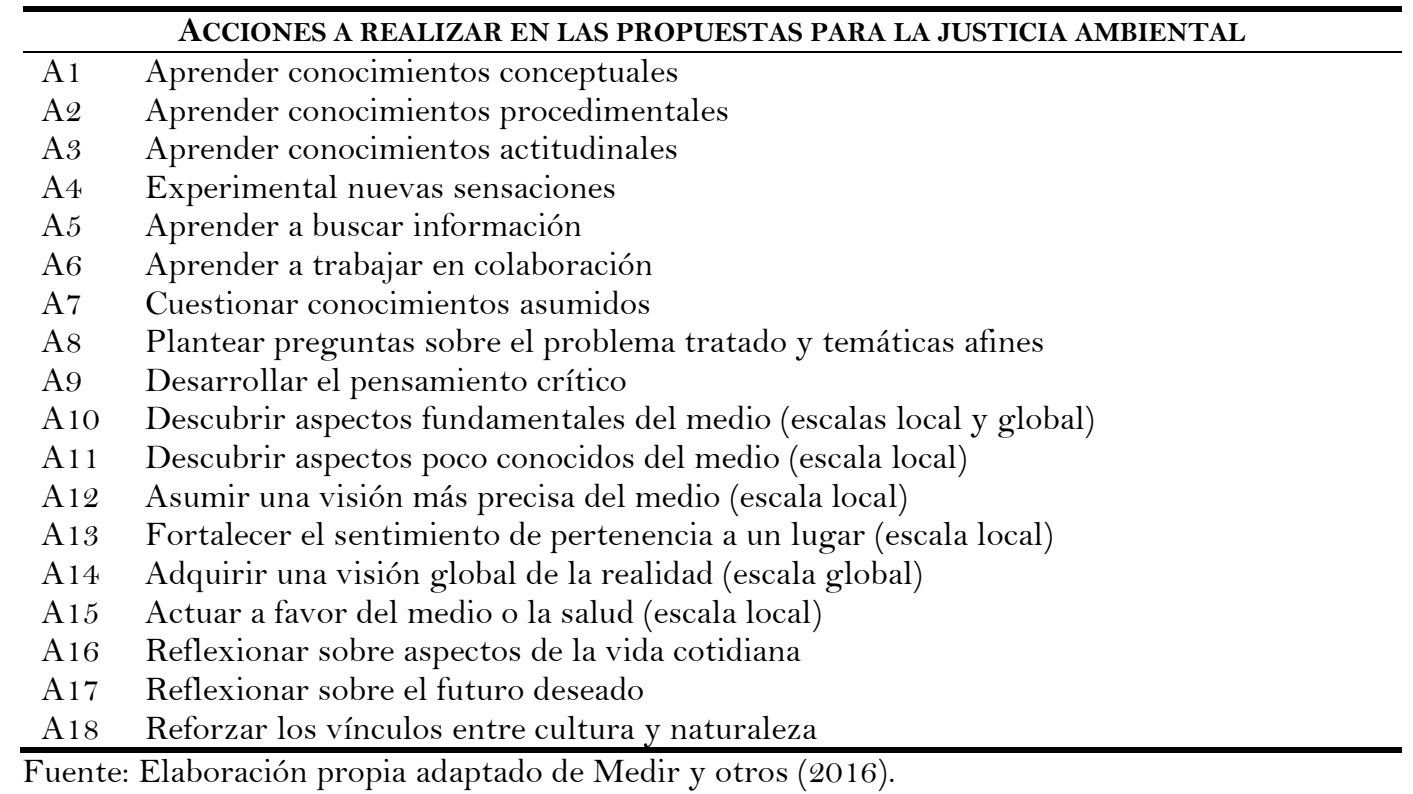

\section{Resultados}

\subsection{Propuesta para educación infantil}

Esta propuesta se realizó en un aula con 51 niños/as de segundo curso del segundo ciclo de Educación Infantil (4-5 años), en un CEIP de la zona sur de Madrid (curso 2016-17). En concreto, se desarrolló una secuencia de actividades sobre la contaminación en el medio marino por residuos sólidos. Esta se llevó a cabo tras un proyecto de aula, titulado "el mar", donde se trabajaron contenidos como los animales marinos, las relaciones tróficas (carnívoro, herbívoro, productor), y los medios de transporte y las profesiones.

La propuesta se estructuró en cuatro bloques: a) presentación de animales marinos extintos, que permitió conectar a los estudiantes con el proyecto de aula mencionado anteriormente; b) diálogo sobre la causa de su desaparición y presentación de animales en peligro de extinción y sus causas (entre ellas la basura generada por el ser humano); c) procesamiento y reciclaje de residuos sólidos, y toma de decisiones sobre cómo podrían reducir su producción; y d) lectura de un cuento sobre la sobreexplotación del medio ambiente y el consumo descontrolado. La duración total fue de una hora y quince minutos, y los contenidos tratados, así como sus relaciones (figura 1).

Reunidos en asamblea los 51 alumnos (pertenecientes a los dos grupos-clase de segundo ciclo) se presentaron casos de animales marinos de gran tamaño como reptiles marinos (mosasaurio, plesiosaurio, ictiosaurio) y peces (placodermos del devónico, megalodón), todos ellos extintos, y que permitían enlazar la fauna prehistórica con los organismos marinos actuales, estudiados en el proyecto anterior.

Una vez explicado cómo podíamos conocer su existencia a través de fósiles, se plantearon las preguntas: “¿Os gustaría haber convivido con ellos?” “Quién querría haber vivido con un megalodón, un tiranosaurio?” Todos querrían haber coexistido con ellos, hasta que algunos indicaron los riesgos: "No, porque nos hubieran comido a todos". Tras su discusión, convinieron que la pérdida de estos animales fue grave para el planeta, pero que 
los seres humanos no tuvimos nada que ver, que fueron cataclismos derivados del impacto de un meteorito.

A continuación, se preguntó “ ¿Cómo os sentiríais si se hubieran extinguido porque los adultos hubiéramos destruido el ambiente en el que vivían?”. Contestaron que estarían enfadados y que ellos los hubieran defendido. Esto sirvió como punto de partida para presentarles imágenes de seres vivos actuales que se encuentran en peligro de extinción y el papel del ser humano en ello. Se seleccionaron ejemplos de organismos que ellos pudieran conocer como pingüinos, tortugas y focas, como se muestra en la presentación (http://bit.ly/2uoHUwk).

Un ejemplo concreto fueron las aves marinas, sobre las que se preguntó: “¿sabéis qué comen las aves marinas?”. Gracias a su proyecto, respondieron correctamente que peces. En ese momento se cuestionó “¿sabéis cómo pescan las aves marinas?”. Dijeron que se lanzaban en picado sobre el agua y que entraban en ella a pescar. La presentación confirmó su respuesta y vieron cómo pescan haciendo vuelos cercanos al agua y cómo los peces que les sirven de alimento utilizan a su vez los nutrientes de la superficie del mar. Del mismo modo preguntamos “¿cómo comen los peces?”. Como habían tenido un pez en clase y le habían dado de comer, explicaron que suben la boca hacia la superficie y comen su comida que son unas hojas de colores pequeñas que venden en la tienda de animales. Finalmente, usamos imágenes reales de la superficie del mar cuando está contaminada con residuos sólidos de plástico, y les mostramos cómo lo ven los peces desde el fondo del mar y las aves en vuelo. De este modo entendieron cómo confunden la basura con la comida. Tras esto, se presentaron distintas imágenes de aves marinas y peces que contenían en su interior restos de materiales de plástico que están contaminando los mares y océanos, así como otros que impiden su normal alimentación y desarrollo. También, aparecieron imágenes de mamíferos marinos y tortugas atrapados en redes o asfixiados por plásticos.

Todo ello puso en evidencia la necesidad de hacer algo para evitarlo, por tanto, se les propuso el reto de ayudar a estos animales. En primer lugar, se preguntó sobre el origen de estos materiales. Reconocieron entre otros, bolsas, botellas, vasos de refresco y redes. Confirmando que se trataba de productos de uso doméstico que ellos utilizaban diariamente. A raíz de esto, reconocieron que la basura procedía de las ciudades, y que todos contribuimos a contaminar el medio marino. Finalmente, acordaron la necesidad de realizar acciones específicas para evitarlo, y un ejemplo fue el siguiente. Llevamos al aula los anillos de plástico que se usan como agrupadores de las latas de refresco (figura 2A), se recordó lo que pasaba con algunos animales, como las tortugas, y se comprobó la resistencia de las anillas. Los alumnos, tras esta comprobación, propusieron que para evitar que los animales quedaran atrapados, debían cortarlos y tirarlos al contenedor amarillo. 


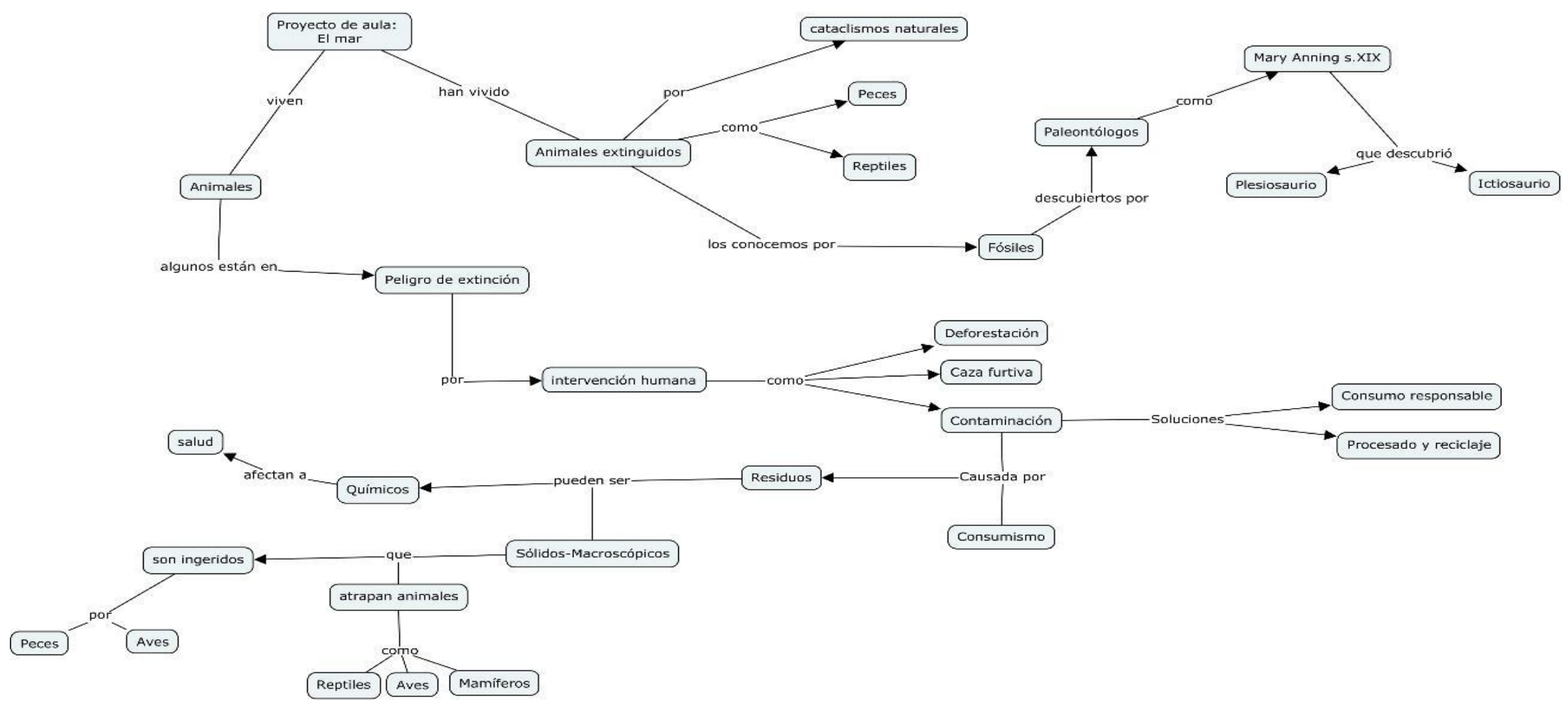

Figura 1. Mapa conceptual de la propuesta desarrollada con los alumnos de educación infantil

Fuente: Elaboración propia. 

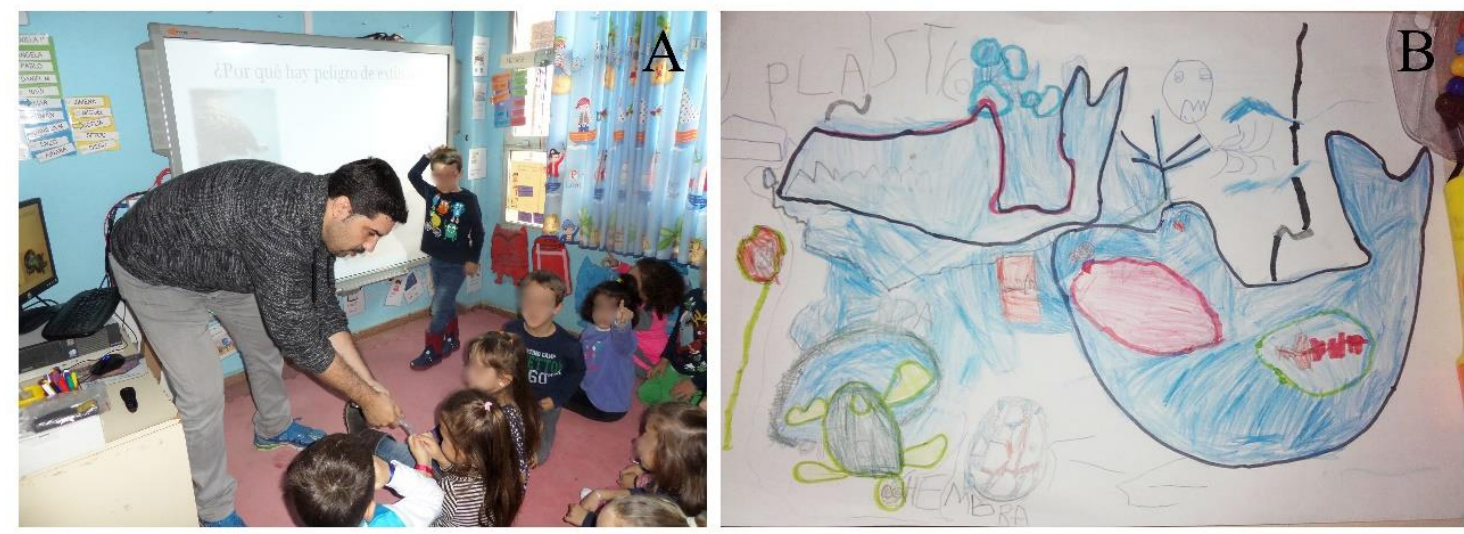

Figura 2. En A, comprobamos la resistencia que tienen los anillos de plástico de las latas de refresco. En B, dibujo infantil de los problemas de contaminación y sus efectos sobre diferentes animales marinos

Fuente: Elaboración propia.

Además, reconocieron que este problema no es exclusivo del medio marino y que el consumo desmesurado conduce a la destrucción de todos los ambientes naturales del planeta, no solo los acuáticos. Por lo que aparte de reciclar, acordaron que era necesario un consumo responsable de cada uno de ellos. Para trabajar en un ecosistema terrestre concreto, como es un bosque, se les contó el cuento "En el bosque del perezoso" (Editorial Hipòtesi). Esto permitió que los alumnos pudieran adquirir una visión global del problema, aplicando lo aprendido en el mar en un nuevo contexto, pero entendiendo que el papel del ser humano en ambos casos es el mismo, y que ellos deben aportar a la cuestión.

Para conocer el aprendizaje del alumnado, las maestras recurrieron a la realización de un dibujo sobre el tema de la contaminación marina. En la figura $2 B$, se muestra el dibujo de uno de los alumnos. En él, representa los problemas que provoca la contaminación en diferentes animales marinos, y la explicación que da sobre su dibujo, es la siguiente: "El tiburón tiene... cosas...plástico de la Coca-cola... en la aleta dorsal", que "La ballena se va a comer una Coca-cola y se ha comido unos guantes de boxeo en una cuerda". También representa que "La tortuga hembra tiene un montón de huevos y está atada a una cuerda y no puede darles de comer". E incluso describe cómo "Al pulpo le pasa que iba a observar ese pincho y se ha acercado mucho que se le ha metido en el ojo". Lo que evidencia es que existe una toma de conciencia del problema de las basuras producidas por los seres humanos que compromete a la supervivencia de los animales. Según el dibujo del alumno, la situación se presenta con alta frecuencia lo que sugiere que existe preocupación por ello.

En el cuadro 2, se muestra un cuadro con las acciones realizadas en cada actividad, donde se aprecian las fases de distribución de contenidos, reflexión sobre lo aprendido y aplicación para resolver problemas (acción).

\subsection{Propuesta para bachillerato}

Esta propuesta se enmarca en las jornadas culturales de un Instituto de Enseñanza Secundaria de la zona sur de Madrid. Participaron 57 alumnos de dos grupos-clase de la asignatura de Biología de $2^{\circ}$ de Bachillerato del itinerario de Ciencias y Tecnología (inicio del tercer trimestre del curso 2010-11). Debido a su programación, ya conocían conceptos relacionados con la célula y comenzaban con los ecosistemas. 
Cuadro 2. Acciones realizadas por los alumnos de educación infantil

\begin{tabular}{llc}
\hline \multicolumn{1}{c}{ FASES } & \multicolumn{1}{c}{ ACTIVIDADES } & ACCIONES \\
\hline Reflexión & Presentación de animales marinos & A8 \\
\hline Distribución & Diálogo sobre la desaparición de especies & $\begin{array}{l}\text { A1, A3, A7, A8, A9, } \\
\text { A10, A11, A12, A17 }\end{array}$ \\
\hline Reflexión y acción & Procesamientos de residuos sólidos & $\begin{array}{l}\text { A2, A6, A7, A8, A9, } \\
\text { A11, A15, A16, A18 }\end{array}$ \\
\hline Distribución & Lectura de un cuento infantil & A14 \\
\hline Fuente: Elaboración propia. &
\end{tabular}

El tema abordado fue la presencia de productos farmacéuticos y de cuidado personal en aguas de uso humano y los efectos que provocan sobre los seres vivos. La propuesta se estructuró en dos bloques: a) presentación de contenidos (50 minutos) y b) diálogo sobre los aspectos más relevantes de la problemática (30 minutos).

La presentación de contenidos se realizó a través de una conferencia titulada: "La Toxicología celular va al instituto: una aproximación multidisciplinar para los estudiantes de Ciencias Naturales de Bachillerato". Se presentó de forma integrada y multidisciplinar diferentes contenidos estudiados en Secundaria y Bachillerato como los ecosistemas, la contaminación industrial, la estructura de la célula, la fisiología humana y la salud. Durante la exposición se establecieron las relaciones existentes entre la contaminación ambiental, su origen y los compartimentos del ecosistema que ocupan los contaminantes. Además, se asociaron las implicaciones del consumo y los vertidos domésticos con la presencia de esas sustancias en el medio y sus efectos para la salud (figura 3).

La exposición fue guiada con preguntas que hacían participar a los asistentes y enlazaban los contenidos que habían estudiado con los que se presentaban. Se inició con la idea de que la industria es el origen de la contaminación ambiental. En este punto, se plantaron las siguientes cuestiones: “¿Diariamente os ducháis?, ¿usáis colonias, desodorantes, algún cosmético, etc.?, ¿consumís fármacos con cierta frecuencia?”. Los alumnos asintieron confirmando que estos hechos ocurrían. Sin embargo, ante la pregunta: “¿Conocéis algún compuesto químico que esté presente en las etiquetas de los productos que usáis?”, no fueron capaces de mencionar ninguno, salvo el de fármacos como paracetamol o ibuprofeno. Se les mostraron listados de compuestos, entre ellos los aditivos, denominados E-XXX. Se explicó que se consideran compuestos inertes sin efectos biológicos, aunque cada vez más estudios demuestran que era incorrecto. Sin embargo, estamos expuestos voluntariamente a ellos, incorporándose una parte a nuestro cuerpo. El resto sale por los desagües domésticos, entrando finalmente en contacto con diferentes seres vivos. A raíz de este hecho, se les cuestionó si consideraban que podrían tener un efecto sobre estos animales y plantas. En general, respondiendo que "si no nos afectan a nosotros, ¿por qué les iba a pasar algo?” o que "Son compuestos seguros si no, no se venderían".

A partir de aquí, se mostraron las tasas de eliminación de estos compuestos en las depuradoras, demostrando que en muchos casos no se eliminan. Se presentaron herramientas de análisis toxicológico para valorar los riesgos para la salud y se les preguntó “QQué enfermedades consideráis más relevantes actualmente?”. 

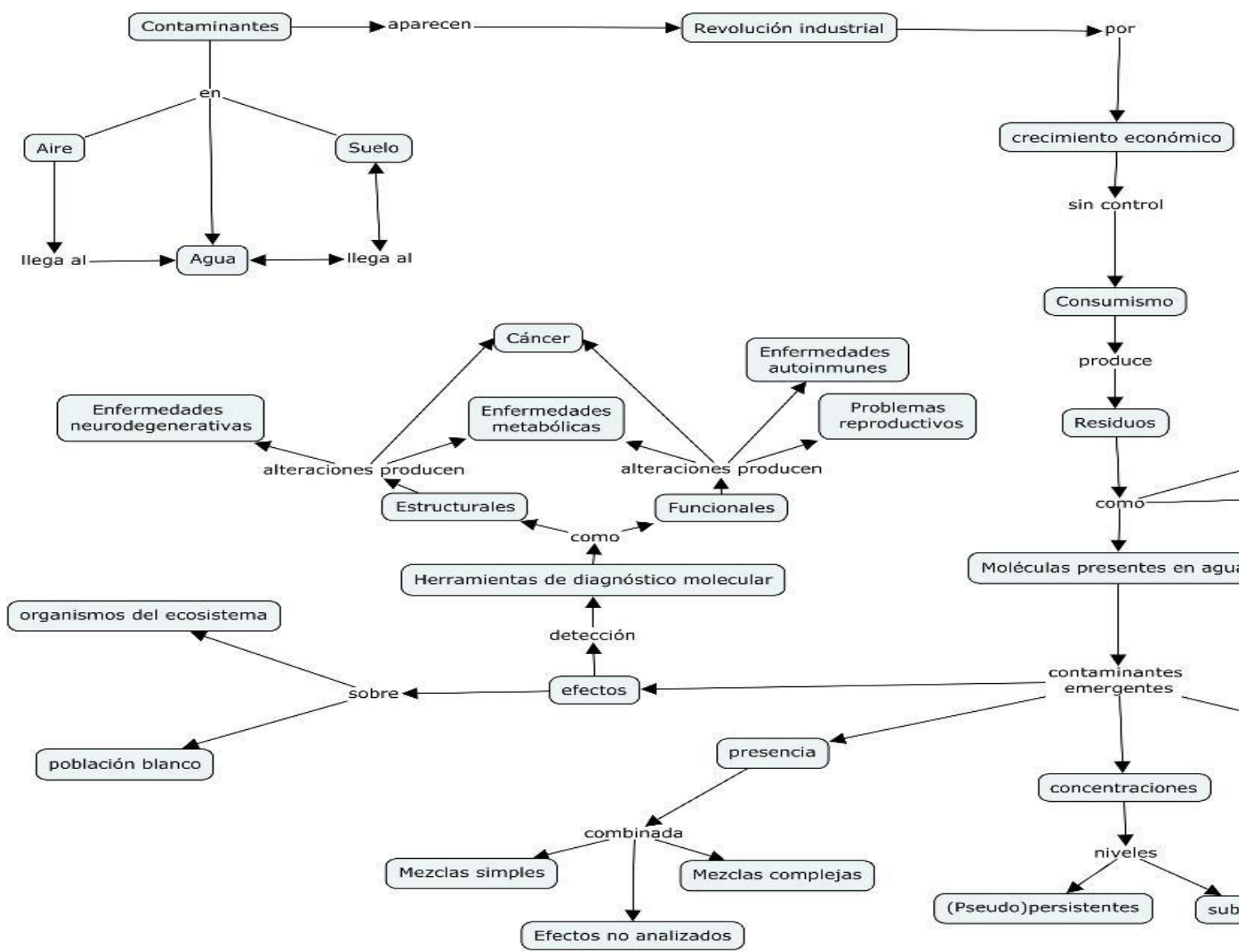

Figura 3. Mapa conceptual de la presentación expuesta a los alumnos de bachillerato

Fuente: Elaboración propia. 
Las primeras respuestas fueron "cáncer" y "tumores". Algunos indicaron: "las malformaciones [de neonatos]”. Estas son las que tradicionalmente se asocian con exposiciones a sustancias químicas, por lo que se insistió, y respondieron: "problemas respiratorios". Otras que se mencionaron fueron las enfermedades de Parkinson y de Alzheimer o las enfermedades del sistema inmunitario (alergias y autoinmunes). Se explicó que toda alteración a nivel celular puede tener una consecuencia a nivel de organismo, y se les mostraron cambios en la estructura de las células expuestas a fármacos y productos de cuidado personal y sus mezclas, relacionando las alteraciones con las disfunciones orgánicas y las enfermedades. Finalmente, se planteó la necesidad de realizar estudios toxicológicos, y que su información fuera distribuida entre los ciudadanos y considerada para establecer regulaciones que garantizaran la salud ambiental y humana.

La segunda parte, se inició con preguntas de los propios alumnos como "si esto es así, cipor qué no se hace algo?, ¿se prohíben estas sustancias?, ¿se construyen depuradoras que sean más eficaces en la eliminación de residuos?". La respuesta dada fue: "los intereses económicos". Se les explicó que como nadie moría fulminado por estos compuestos, el establecimiento de las relaciones entre causa y efecto se alargaba en el tiempo y era complejo demostrar las relaciones que en un laboratorio se podían determinar. Sustituir o eliminar una sustancia del mercado supone un coste elevadísimo para la industria. Sin embargo, se les mostró el caso de los parabenos en 2004 (Concentrations of parabens in human breast tumours) que generó alarma social y cambió la forma de consumir de los ciudadanos, conduciendo a su sustitución en algunos productos (http://bit.ly/2vopXy4), aunque se siguen utilizando actualmente (http://bit.ly/2nEPWwb). Con este caso, se pretendía que reconocieran que si se convierten en ciudadanos alfabetizados científicamente pueden tomar decisiones que cambien la sociedad.

La cuestión sobre la planta depuradora, tuvo aún más relevancia por su proximidad, ya que este centro está situado en una ciudad con esta infraestructura, que se puede ver " $\mathrm{y}$ oler" como decían ellos, en los alrededores de un parque natural que tiene el municipio. Según sus explicaciones, consideraban que "la depuradora es un filtro [infranqueable] para sustancias tóxicas y hace discurrir el agua limpia”. Por ello, tras su depuración, el curso de agua no debería suponer ningún peligro para las especies de la zona. Se sugirió que visitaran el arroyo en el que vierte la depuradora y que valorasen su estado. Algunos conocían la zona, decían que "olía muy mal" y que "las plantas tenían un aspecto mustio, sucio", “y a veces una capa de espuma blanquecina”. Todo ello generó un estado de inquietud ante la eficacia en la eliminación parcial de los residuos. Valorando como posible solución "reducir el uso de estos productos [cosméticos] y así verter menos por los desagües domésticos". Otros planteaban que "si los productos fuesen naturales y biodegradables, no habría estos problemas", y otros alumnos decían que "esos productos no eran igual de buenos [duraderos]" o "eran demasiado caros". Con lo que volvíamos a la problemática socio-económica para la reducción de los vertidos de origen doméstico. Algunos comentaron que "esta información debería ser publicada en telediarios y prensa. Porque, aunque lo conozcan algunos ciudadanos, la presión que se puede hacer a las empresas es muy pequeña". Además, añadían que "muchas veces yo no hago las compras. Es mi madre que como no sabe esto, sólo mira calidad-precio".

En el cuadro 3 se presentan las acciones realizadas en cada actividad, distinguiéndose una fase de distribución de contenidos y otra de reflexión y propuesta de acción sobre lo presentado. 
Cuadro 3. Acciones realizadas por los alumnos de bachillerato

\begin{tabular}{ccc}
\hline \multicolumn{1}{c}{ FASES } & \multicolumn{1}{c}{ ACTIVIDADES } & ACCIONES \\
\hline Distribución & Presentación sobre medio ambiente y salud & A1, A2, A3, A7, A11 \\
\hline Reflexión y acción & $\begin{array}{c}\text { Diálogo sobre la presencia de contaminantes y } \\
\text { sus efectos }\end{array}$ & $\begin{array}{c}\text { A7, A8, A9, A12, A15, } \\
\text { A16, A17 }\end{array}$ \\
\hline
\end{tabular}

Fuente: Elaboración propia.

\subsection{Propuesta para educación superior}

En este último apartado, nos centramos en la propuesta realizada con dos grupos de alumnos (162) de la asignatura de Prevención y Promoción de la Salud escolar del Grado en Educación Infantil de la Universidad Camilo José Cela en el curso 2015-16. Se trata de alumnos que acceden al grado desde titulaciones de Educación Primaria y su formación en Ciencias es relativamente baja, al proceder mayoritariamente de Humanidades y Ciencias Sociales.

El tema tratado fue la presencia de contaminantes ambientales y sustancias de uso doméstico e industrial en los alimentos, qué vías de exposición existen, y cómo se relacionan con problemas de salud infantil como la obesidad. La propuesta se distribuyó en tres bloques: a) presentación de la problemática ( 1 hora), b) diálogo sobre puntos de interés (30 minutos) y c) elaboración de una hipotética circular en la que se informara a los padres del problema, dándoles recursos sobre el tema.

La presentación utilizada está disponible en un blog creado para la difusión de esta temática (http:// toxicologiacelular.blogspot.com.es/). En ella se describen los problemas de contaminación en los diferentes compartimentos del ecosistema (aire, agua, suelos y organismos) y cómo llegan los compuestos químicos hasta nosotros a través de los alimentos. Además, se mencionan las alteraciones de la salud inducidas por la presencia de estas sustancias. Las dosis bajas y el metabolismo nos ayudan a su eliminación, pero la exposición continua supone un estrés que nos pone en peligro. Dicha situación sigue sin tener normativas que puedan valorar los efectos sobre la salud. Sin embargo, múltiples estudios proponen que este contexto es el responsable de problemas reproductivos e inmunitarios o cáncer, entre otros. Los conceptos presentados y sus relaciones se muestran en la figura 4.

La presentación comienza con la contaminación en los diferentes compartimentos ambientales y se pregunta sobre los diferentes organismos que sirven de alimentos para los seres humanos. Empezamos por el suelo y las respuestas son verduras, frutas y animales de granja. En ese momento se les pregunta sobre la nutrición vegetal, “¡sabéis de dónde toman los nutrientes las plantas?”. Algunos responden que "del suelo", otros añaden que "por las raíces" o que "se absorben sustancias a través de las hojas". En ese punto les cuestionamos "qué nutrientes toman", a lo que responden que "agua y sales minerales", incluso añaden "dióxido de carbono". Se muestran ejemplos de los niveles de insecticidas, herbicidas y metales pesados que se acumulan en plantas cuando el suelo está contaminado. 


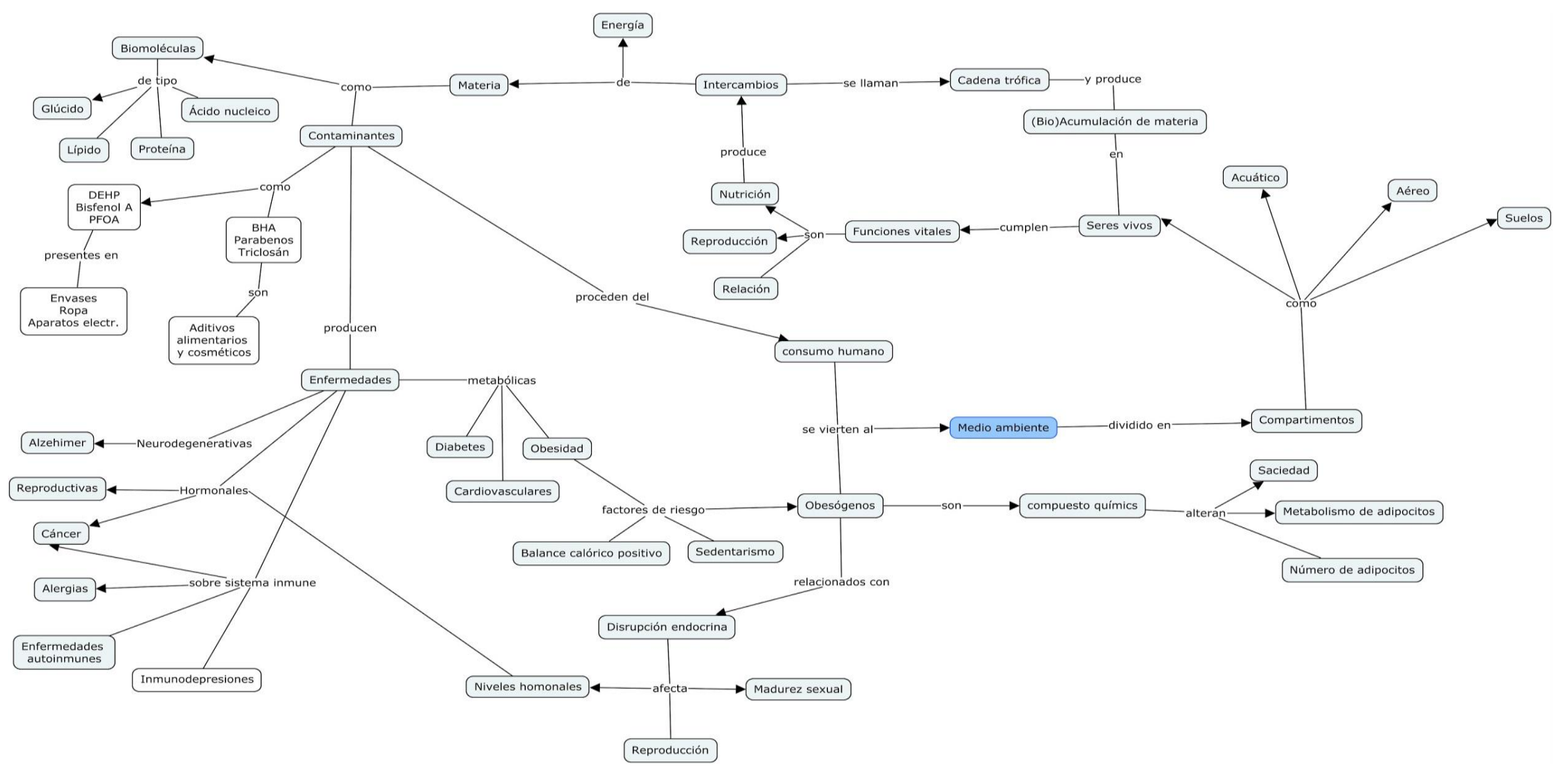

Figura 4. Mapa conceptual y relaciones entre las ideas presentadas en educación superior

Nota: Debido a la multitud de relaciones existentes, se generan bucles, y por ello recomendamos la idea "Medio ambiente" marcado en azul como el inicio de este mapa. Fuente: Elaboración propia. 
Explicando que, cuando las plantas toman del sustrato agua y sales minerales, también se incorporan estas sustancias. Por lo tanto, si la planta lo incorpora, “¿qué pasará cuando la comemos?". La respuesta fue "que nos la comemos". En ese punto algunos alumnos sugerían que "el uso de pesticidas, herbicidas y abonos naturales eran la solución, porque no había químicos". Les explicamos que probablemente reducía la presencia de algunos compuestos, pero les mostramos que los compuestos químicos pueden ser tóxicos incluso siendo de origen natural y les introdujimos la siguiente pregunta: "la producción ganadera intensiva, ¿dónde se produce?”, a lo que contestaron que "en la granja”. Les planteamos dos palabras: establo y pocilga, y les pedimos que nos indicasen qué les sugería. "Suciedad", respondieron inmediatamente. En ese punto, se explicó que el hacinamiento permite abaratar costes de producción, pero que requiere de cuidados extras para evitar enfermedades, y que suelen tratar a los animales con antibióticos, que se excretan y también se incorporan a sus tejidos. De manera que ni el abono ganadero está limpio de químicos, ni la carne tampoco. Además, se señaló que esta exposición continua es lo que pone en peligro el uso de antibióticos en el futuro, debido a las resistencias bacterianas. Se explicó también que los antibióticos han tenido papel en el engorde de los animales y que se ha descrito el mismo efecto en niños.

A continuación, se mostró el mar, y se preguntó por los alimentos que consumen de allí. Las respuestas fueron pescados y mariscos. Posteriormente se utilizaron imágenes del mar contaminado con vertidos y residuos, y se indicó que hay otros que no se ven, pero que también están, como vertidos de origen doméstico, fármacos y productos de cuidado personal. Les preguntamos “¿conocéis la enfermedad de Minamata?”, ninguno la conocía. “¿Conocéis qué es el metil-mercurio?”, tampoco lo conocían. La presentación les mostró las alteraciones neurológicas sufridas en Japón en los años 50 y 60 por comer pescado contaminado por metil-mercurio. El vertido industrial afectó a miles de personas. Las siguientes preguntas fueron: “Sabéis que los pescados depredadores que consumimos en España también están contaminados con metil-mercurio?”, “¿y que las autoridades sanitarias no lo consideran apto para consumo infantil ni mujeres embarazadas o lactantes?”. La primera respuesta fue otra pregunta, “¿para el resto sí?” y ante la respuesta afirmativa, la siguiente: “¿por qué?”. Se respondió que “el motivo es que se considera muy peligroso para individuos en desarrollo, aunque restringen su consumo hasta la edad de 12 años a $50 \mathrm{~g} /$ semana de estos peces”. En esta misma línea se cuestionó sobre “¿quién consume el interior de las cabezas de las gambas?”, ya que también acumulan metales pesados y son transferidos a nuestro cuerpo. A partir de aquí se abordaron los envases y su reciclaje para evitar la contaminación, pero preguntamos: “`sabíais que en los alimentos quedan restos de estos plastificantes y sus aditivos?”. Se mencionaron algunos como los ftalatos y los bisfenoles y les preguntamos por sus alimentos preferidos, embutidos, queso, huevos, etc. Luego se revisó la presencia de estos compuestos en ellos, demostrando que es un problema que les afecta en su día a día. A partir de ahí, se explicó la asociación de la exposición a estos compuestos con enfermedades como cáncer, diabetes y obesidad.

Se preguntó si conocían la feminización de las poblaciones naturales de moluscos, peces, anfibios e incluso osos polares. Les sorprendió el hecho de que se pudieran dar estas situaciones por los contaminantes ambientales. Se comentó su presencia en fármacos (píldoras anticonceptivas y tratamientos antitumorales), ya que interaccionan con receptores hormonales e impiden su función correcta. Compuestos como el bisfenol A o los antiadherentes (teflón, concretamente PFOA) comprometen nuestra salud poniendo en peligro nuestro sistema hormonal, la reproducción, induciendo cáncer y enfermedades 
como dislipemias. Para acabar, les preguntamos “¿conocéis algún producto químico que provoque el consumo desmesurado y sin control?”, "si lo hubiera, ¿qué efectos tendría?”. No conocían el nombre, pero algunos decían que habían "oído hablar sobre ese tema" y que "los fabricantes lo ponían en los snacks". Mencionamos el caso del glutamato monosódico y cómo altera la señalización para afectar a la saciedad, induciendo el consumo de alimentos sin control.

Tras este bloque, se inició un diálogo en el que observamos la sorpresa por la presencia de sustancias químicas en alimentos y sus potenciales riesgos para la salud. Los participantes insistían en que "estas informaciones no llegaban a las vías de comunicación convencionales como la televisión y los periódicos" y decían que "probablemente se quedaban en foros académicos". Por lo tanto "la toma de decisiones en el día a día se hacía sin tener en cuenta esta información". Ante su respuesta, se mostró que en medios de comunicación había noticias que hablaban del tema, pero que el público apenas les prestaba atención. Ellos decían que "si fuera importante, y la salud lo es, deberían informar de ello con más énfasis y no en artículos de prensa en páginas interiores". Incluso algunos las comparaban con otras como "si un futbolista juega mejor o peor, o ficha por un equipo u otro". Concluyendo que conocer estas informaciones proporciona criterio para la toma de decisiones y que "quizás no interese al poder económico que se conozcan estos temas". También comentaron que "el ministerio de sanidad debería notificar estas cuestiones a la población" de forma más garantista, se mencionaron casos concretos como "la recomendación médica de introducir, por ejemplo, las acelgas en los purés de los bebés, cuando son alimentos que acumulan porquerías".

Para terminar, se solicitó elaborar una circular en que hipotéticamente debían informar a los padres de la problemática tratada en el aula. Para su construcción debían decidir qué información consideraban más relevante para el ambiente escolar, y cuál era la mejor forma de presentarla. Estas circulares fueron entregadas a los docentes, y en su análisis se encontró que los futuros maestros fueron capaces de realizar un ejercicio de síntesis excelente. Las cartas mostraron apartados sobre la presencia de tóxicos, fuentes de exposición, usos domésticos, efectos para la salud, fuentes de información (conferencias en vídeo, noticias y webs). Cabe reseñar que los alumnos destacan los obesógenos y su implicación en la obesidad, así como una parte final en todas cartas con propuestas de acción domésticas para reducir la exposición. Algunos ejemplos son las siguientes:

Desde el colegio os animamos a que reflexionéis sobre el contenido de esta circular y colaboréis en la salud de vuestro hijos con hechos tan simples como: sustituir las botellas de plástico por termos de aluminio, limitar el uso de comidas basura, intentar aumentar el consumo de frutas y verduras orgánicas que han estado menos expuestas a los pesticidas, evitar el abuso de chucherías, evitar calentar comidas en el microondas en envases de plástico, reducir el número de horas que los niños/as pasan delante de la tele y evitar el sedentarismo, entre otras. (RMBR)

Os damos algunas recomendaciones sencillas: no meter plásticos en el microondas, no rascar con objetos metálicos las sartenes de teflón, instalar filtros de carbón en los grifos si bebéis agua de la red, comprar las carnes en el mostrador de frescos y no en la de envasados, consumir pescados como el salmón (rico en ácidos grasos Omega-3) y carnes sin antibióticos, reducir el consumo de alimentos enlatados o sustituir los envases plásticos (agua mineral, refrescos, etc.) por los de cristal o aluminio. (IRR)

En el cuadro 4 se presenta un resumen de las acciones realizadas en cada actividad. Se puede distinguir claramente una fase de distribución de contenidos, otra de reflexión sobre lo aprendido y, por último, en la que proponen formas de actuación. 
Cuadro 4. Acciones puestas en práctica por los alumnos de educación superior

\begin{tabular}{lcc}
\hline \multicolumn{1}{c}{ FASES } & ACTIVIDADES & ACCIONES \\
\hline \multirow{2}{*}{ Distribución } & $\begin{array}{c}\text { Presentación sobre medio ambiente, } \\
\text { alimentación y salud }\end{array}$ & $\begin{array}{c}\text { A1, A2, A3, A9, A10, } \\
\text { A11 }\end{array}$ \\
\hline \multirow{2}{*}{ Reflexión } & $\begin{array}{c}\text { Diálogo sobre la presencia de contaminantes en } \\
\text { alimentos y sus efectos }\end{array}$ & $\begin{array}{c}\text { A2, A3, A4, A7, A8, } \\
\text { A9, A12, A13, A15, } \\
\text { A16, A17 }\end{array}$ \\
\hline \multirow{2}{*}{ Acción } & Elaboración de la circular escolar & A5, A12, A15, A16, \\
& & A17 \\
\hline
\end{tabular}

Fuente: Elaboración propia.

\subsection{Análisis conjunto de las experiencias}

En conjunto, las experiencias descritas aquí muestran resultados sobre acciones de interés en la alfabetización científica con respecto al medio ambiente, que ayudan a poner en práctica una educación ambiental que resulta un elemento clave en la Educación para la justicia social. A pesar de tratarse de niveles educativos muy diferentes, en todos los casos se distribuyeron contenidos adaptados al curso correspondiente, así como de interés para ellos. Siempre poniendo en práctica las acciones A1, A3, A11, y las acciones A2, A7, A8, A9 y A10 en al menos dos de los niveles académicos.

En las etapas de reflexión de cada una de las intervenciones realizadas, siempre se pusieron en práctica las acciones A7, A8, A12, A15 y A16, que están involucradas en la acción A9 (pensamiento crítico) que también se cubrió para el contexto de cada actividad. Por lo tanto, la fase de reflexión se inducía mediante preguntas mediadoras que permitieron asociar los contenidos presentados con los de su vida cotidiana y con las problemáticas, ayudando a despertar su sentido crítico.

A partir de la reflexión y el cuestionamiento de la realidad y sus complejidades en el contexto de la educación ambiental, los alumnos de los tres niveles propusieron acciones para cambiar la situación, que siempre estuvieron ligadas a la protección de la salud y del medio ambiente (A 15), y en los niveles académicos más elevados también relacionados con el futuro deseado (A17).

\section{Discusión y conclusiones}

Generalmente, la educación ambiental se ha centrado fundamentalmente en acciones de tipo conceptual, presentando los problemas del entorno, ya que en muchas ocasiones la sociedad las desconoce. Sin embargo, se necesitan propuestas que desarrollen también destrezas actitudinales y procedimentales (Medir et al., 2016; Novo, 2005), que están en consonancia con el desarrollo de las destrezas científicas (de Pro, 2013) y los principios de CTSA (Vilches et al., 2008). Además, desde hace mucho tiempo múltiples estudios indican que existe interés y conciencia ambiental en diferentes colectivos (Benegas y Marcén, 1995; Cantó, 2016; García-Esteban y Murga-Menoyo, 2015). Sin embargo, en ocasiones, sus conductas diarias no lo reflejan, debido a que se requieren acciones cotidianas que suponen esfuerzos cuyo incumplimiento no tiene repercusión inmediata en su día a día, porque son problemas globales y alejados de su entorno próximo (Benegas y Marcén, 1995). Además, por lo general, tratan de mostrar la asociación de un problema con una solución, sin requerir de la participación de los ciudadanos en la generación de soluciones. 
Por ello, nuestras propuestas tratan la contaminación y su repercusión sobre los seres vivos a diferentes escalas, adaptándolas a los centros de interés de cada colectivo, pero sobre todo haciendo partícipes a los alumnos de la implementación de las soluciones que existen y la búsqueda de nuevas. Para lograrlo, se trabaja desde una perspectiva integral, presentando los motivos que tiene la sociedad (sociales, éticas, económicas, científicas, ambientalistas, etc.) para mantener o eliminar esas situaciones, por lo que creemos que sí podemos tener un mayor impacto sobre cambios de actitudes ambientales.

Para su consecución, consideramos clave, el paso que deben dar los alumnos entre la distribución de los conocimientos y la participación en la toma de decisiones, que es la capacitación en destrezas científicas, como búsqueda de información, cuestionamiento de lo aprendido y desarrollo de pensamiento crítico. Por ello, en las experiencias presentadas, siempre ha existido una fase de reflexión tras las acciones distributivas. En ellas, mediante preguntas que guiaban, o que surgían del propio colectivo, los alumnos han tomado una posición activa, proponiendo soluciones o acciones personales, conduciéndoles a la fase de acción.

Sin embargo, es muy complejo estimar si estos cambios de actitud desencadenan cambios de conducta o comportamientos a largo plazo. Hay que destacar que generalmente este tipo de intervenciones ponen conceptualmente a todos de acuerdo, ya que proteger el medio ambiente y nuestra salud es algo que nos une a todos. Además, en estas intervenciones que aquí presentamos hay una idea más, es el principio de autoridad. Un experto en el tema aparece en clase para contar algo que poca gente en la sala conoce en profundidad, quizás esto también aporte cierto sesgo en la recogida de respuestas, aunque a nuestro juicio no pareció influir en los resultados obtenidos, sobre todo en los niveles académicos más bajos. En conjunto podemos decir que las intervenciones impactaron en los alumnos. Por ello la consideramos efectiva en la distribución de los contenidos, en la reflexión colectiva y, al menos en el corto plazo, en el despertar de la conciencia ambiental, aunque no se pueden juzgar cambios en la conducta real en su vida cotidiana.

En este sentido, las acciones más destacadas desarrolladas en nuestras experiencias de aula son el aprendizaje de conocimientos actitudinales, el cuestionamiento de conocimientos asumidos, el planteamiento de preguntas sobre el tema, el desarrollo del pensamiento crítico, las actuaciones a favor del medio o de la salud y las reflexiones sobre aspectos de la vida cotidiana y del futuro deseado. Acciones que se considera que no están siendo debidamente implementadas y que aún deben ser trabajadas en los programas de Educación Ambiental (Medir et al., 2016).

Por último, las propuestas presentadas podrían suponer una mejora educativa, dado que abordan la problemática de la carencia de acciones medioambientales para la Educación Infantil (Davis, 2009; Elliott, 2010), así como para la formación de profesorado (Cantó, 2016; García-Esteban y Murga-Menoyo, 2015; Solís-Espallargas y ValderramaHernández, 2015), desde su papel como docentes y ciudadanos. Además, debemos considerar que los colectivos de población no adulta (Educación Infantil y Bachillerato) deben posicionarse en estos temas, ya que tienen mucho que aportar desde la perspectiva de justicia ambiental como recogen diferentes legislaciones internacionales, donde se les da derecho a participar en la toma de decisiones (Derr, 2017). Por lo tanto, consideramos que el enfoque CTSA en la enseñanza de las Ciencias constituye un elemento clave en el desarrollo de una Educación para la justicia ambiental que provoque cambios para un futuro más sostenible. 


\section{Referencias}

Agyerman, J. (2007). Environmental justice and sustainability. En G. Atkinson, S. Dietz y E. Neumayer (Eds.), Handbook of sustainable development (pp. 171-188). Cheltenham: Edward Elgar. https://doi.org/10.4337/9781847205223.00020

Aikenhead, G. S. (2003). STS education: A rose by any other name. En R. Cross (Ed.), Vision for science education: Responding to the work of Peter J. Fensham (pp. 59-75). Nueva York, NY: Routledge Press.

Ainsworth, S., Prain, V. y Tyler, R. (2011). Drawing to learn in science. Science, 333, 1096-1097. https://doi.org/10.1126/science.1204153

Andersson, K. y Gullberg, A. (2014). What is science in preschool and what do teachers have to know to empower children? Cultural Studies of Science Education, 9(2), 275-296. https://doi.org/10.1007/s1 1422-012-9439-6

Arriaga, A. y Pardo, M. (2011). Justicia ambiental. El estado de la cuestión. Revista Internacional de Sociología, 69(3), 627-648. https://doi.org/10.3989/ris.2009.12.210

Benegas, J. y Marcén, C. (1995). La educación ambiental como desencadenante del cambio de actitudes ambientales. Revista Complutense de Educación, 6(2), 11-28.

Bonil, J., Junyent, M. y Pujol, R. M. (2010). Educación para la sostenibilidad desde la perspectiva de la complejidad. Revista Eureka sobre Enseñanza y Divulgación de las Ciencias, 7, 198-215. https://doi.org/10.25267/Rev_Eureka_ensen_divulg_cienc.2010.v7.iextra.05

Cantó, J. (2016). Percepción de la sostenibilidad en los maestros en formación de educación infantil. Indagatio Didactica, 8(1), 96-109.

Cebrián, G. y Junyent, M. (2014). Competencias profesionales en educación para la sostenibilidad: Un estudio exploratorio de la visión de futuros maestros. Enseñanza de las Ciencias, 32(1), 29-49. https://doi.org/10.5565/rev/ensciencias. 877

Chrispino, A., Silva de Lima, L., Bengio, M., Carvalho, A. C. y Ferreira, M. A. (2013). A área CTS no Brasil vista como rede social: ¿Dónde aprendemos? Ciência \& Educação, 19(2), 455-479. https://doi.org/10.1590/S1516-73132013000200015

Davis, J. M. (2009). Revealing the research hole of early childhood education for sustainability: A preliminary survey of the literature. Environmental Education Research, 15(2), 227-241. https://doi.org/10.1080/13504620802710607

Derr, V. (2017). Participation as a supportive framework for cultural inclusion and environmental justice. Revista Internacional de Educación para la Justicia Social (RIEJS), 6(1), 77-89. https://doi.org/10.15366/riejs2017.6.1.004

Dobson, A. (1998). Justice and the environment. Conceptions of environmental sustainability and dimensions of social justice. Oxford: Oxford University Press. https://doi.org/10.1093/0198294956.001.0001

Elliott, S. (2010). Essential, not optional: Education for sustainability in early childhood centers. Education for Sustainability Exchange Magazine, 2, 34-37.

Ezquerra, A. y Fernández, B. (2014). Análisis del contenido científico de la publicidad en la prensa escrita. Revista Eureka sobre Enseñanza y Divulgación de las Ciencias, 11(3), 275-289. https://doi.org/10.25267/Rev_Eureka_ensen_divulg_cienc.2014.v11.i3.01

Fuente, M. E., Tagle, D. y Hernández, E. (2015). La justicia ambiental como atributo del ecosocialismo. Exploraciones teóricas y praxis comunitarias en la gestión del agua. Revista THEOMAI. Estudios Críticos sobre Sociedad y Desarrollo, 32, 170-188. 
García-Esteban, F. E. y Murga-Menoyo, M. A. (2015). El profesorado de educación infantil ante el desarrollo sostenible. Necesidades formativas. Enseñanza \& Teaching, 33(1), 121-142. https://doi.org/10.14201/et2015331121142

García González, S. y Pérez Martín, J. M. (2016). Enseñanza de las ciencias naturales en educación primaria a través de cuentos y preguntas mediadoras. Revista Internacional de Investigación e Innovación en Didáctica de las Humanidades y las Ciencias, 3, 101-122.

Goodnow, J. (1979). El dibujo infantil. Madrid: Morata.

Grass, R. (1995). Environmental education and environmental justice: A three circles perspective. Pathways to Outdoor Communication, 5, 9-13.

Hiskes, R. (2006). Environmental human rights and intergenerational justice. Human Rights Review, 7(3), 81-95. https://doi.org/10.1007/s12142-006-1023-6

Márquez, C., Bonil, J. y Pujol, R. M. (2005). Las preguntas mediadoras como recursos para favorecer la construcción de modelos científicos complejos. Enseñanza de las Ciencias, 23, 15 .

Márquez, C. y Roca, M. (2006). Plantear preguntas: Un punto de partida para aprender ciencias. Revista Educación y Pedagogía, XVIII(45), 61-71.

Martínez, C. y García, S. (2003). Las actividades de primaria y ESO incluidas en los textos escolares. ¿Qué objetivos persiguen? ¿Qué procedimientos enseñan? Enseñanza de las Ciencias, 21(1), 243-264.

Medir, R. M., Heras, R. y Magin, C. (2016). Una propuesta evaluativa para actividades de educación ambiental para la sostenibilidad. Educación XX1, 19(1), 331-355.

Mogesen, F. y Mayer, M. (2009). Perspectivas sobre la educación ambiental. Un marco de trabajo crítico. En F. Mongensen, M. Mayer, S. Breiting y A. Narga (Coords.), Educación para el desarrollo sostenible. Tendencias, divergencias y criterios de calidad (pp. 21-42). Barcelona: Graó.

Murillo, F. J. y Hernández-Castilla, R. (2011). Hacia un concepto de justicia social. REICE. Revista Iberoamericana sobre Calidad, Eficacia y Cambio en Educación, 9(4), 8-23.

Novo, M. (2005). Educación ambiental y educación no formal: Dos realidades que se realimentan. Revista de Educación, 338, 145-165.

Ponce, D. (2012). Procuración y acceso a la justicia ambiental y territorial en México. Publicación Electrónica Instituto de Investigaciones Jurídicas, 6, 111-124.

de Pro, A. (2013). Enseñar procedimientos: por qué y para qué. Alambique. Didáctica de las Ciencias Experimentales, 73, 69-76.

Ramírez, S. J., Galindo, M. G. y Contreras, C. (2015). Justicia ambiental. Entre la utopía y la realidad social. Culturales, 3(1), 225-250.

Riechman, J. (2003). Tres principios básicos de justicia ambiental. Revista Internacional de Filosofía Política, 21, 103-120.

Santos, W. y Mortimer, E. (2009). Abordagem de aspectos sociocientíficos em aulas de ciências: Possibilidades e desafios. Investigações em Ensino de Ciências, 14(2), 191-218.

Sarokin, D. y Schulkin, J. (1994). Environmental justice: Coevolution of environmental concerns and social justice. The Environmentalist, 14(2), 121-129. https://doi.org/10.1007/BFO1901305

Solís-Espallargas, C. y Valderrama-Hernández, R. (2015). La educación para la sostenibilidad en la formación de profesorado. ¿Qué estamos haciendo? Foro de Educación, 13(19), 165-192. https://doi.org/10.14516/fde.2015.013.019.008 
Strieder, R. B. (2012). Abordagens CTS na educação científica no Brasil: Sentidos e perspectivas (Tesis doctoral). Universidade de São Paulo: São Paulo.

Strieder, R. B., Bravo-Torija, B. y Gil-Quílez, M. J. (2017). Ciencia-tecnología-sociedad: ¿Qué estamos haciendo en el ámbito de la investigación en educación en ciencias? Enseñanza de las Ciencias, 35(3), 29-49. https://doi.org/10.5565/rev/ensciencias.2232

Teixeira, M.S. y Formenton, R. (2012). Fontes alternativas de energia automotiva no ensino médio profissionalizante: Análise de uma proposta contextualizada de ensino de física em um curso técnico. Alexandria, 5(1), 33-61.

Viegas, J. y Santos, W. (2012). Ensino de ciências no ensino fundamental por meio de temas sociocientíficos: Análise de uma prática pedagógica com vista à superação do ensino disciplinar. Ciência \& Educação, 18(4), 787-802. https://doi.org/10.1590/S151673132012000400004

Vilches, A., Gil-Pérez, D., Toscano, J. C. y Macías, O. (2008). Obstáculos que pueden estar impidiendo la implicación de la ciudadanía y, en particular, de los educadores, en la construcción de un futuro sostenible. Formas de superarlos. Revista Iberoamericana de Ciencia, Tecnología y Sociedad, $11(4), 139-172$.

Vílchez López, J. E. (2005). El reto de la educación medioambiental en la formación del profesorado de educación primaria. Muestrario de actividades. Escuela Abierta, 8, 97-128.

Wende, N. (2013). CAFOS and environmental justice. The case of North Carolina. Environmental Health Perspectives, 121(6), 182-189. https://doi.org/10.1289/ehp.121-a182

\section{Breve CV de los autores}

\section{José Manuel Pérez Martín}

Doctor en Biología y Licenciado en Biología por la Universidad Autónoma de Madrid (UAM). Profesor del Departamento de Didácticas Específicas de la UAM (ciencias experimentales). Anteriormente ha sido profesor en las facultades de Ciencias de la UAM y de Educación de la Universidad de Camilo José Cela y de la Universidad Internacional de la Rioja (UNIR). Ha impartido docencia en los grados de Biología, Bioquímica, Educación Infantil y Primaria y en másteres de la facultad de Ciencias de la UAM y de Educación de la UNIR. Ha participado en proyectos nacionales y es autor de publicaciones científicas sobre los efectos tóxicos de contaminantes ambientales para la salud humana y ambiental, así como de la enseñanza de las ciencias en revistas nacionales e internacionales indexadas en bases de datos de alto impacto. ORCID ID: 0000-0002-0658-9050. Email: josemanuel.perez@uam.es

\section{Beatriz Bravo Torija}

Doctora en Didáctica de las Ciencias Experimentales y Licenciada en Biología por la Universidad de Santiago de Compostela (USC). Profesora en el área de didáctica de las ciencias experimentales en el Departamento de Didácticas Específicas de la Universidad Autónoma de Madrid. Anteriormente ha sido profesora en las facultades de educación de la Universidad de Granada y de la Universidad de Zaragoza. Ha impartido docencia en los grados de Educación Infantil y Primaria y en el máster de Educación secundaria. Ha participado en proyectos nacionales e internacionales de los que derivan publicaciones científicas sobre la argumentación en problemas sociocientíficos en revistas nacionales e 
internacionales indexadas en bases de datos de alto impacto. ORCID ID: 0000-0001-62366807. Email: beatriz.bravo@uam.es 